\title{
The form and function of post-conflict interactions between female baboons
}

\author{
JOAN B. SILK*, DOROTHY L. CHENEY† \& ROBERT M. SEYFARTH \\ *D epartment of A nthropology, U niversity of C alifornia at $L$ os A ngeles \\ $\dagger D$ epartment of Biology, U niversity of Pennsylvania \\ $\ddagger D$ epartment of P sychology, U niversity of P ennsylvania \\ ( Received 24 M ay 1995; initial acceptance 280 ctober 1995; \\ final acceptance 10 N ovember 1995; M S. number: A7335)
}

\begin{abstract}
A bstract. In many primate species, former opponents engage in friendly behaviours after aggressive conflicts. These kinds of interactions are labelled reconciliation because they are thought to repair relationships damaged by conflicts and help to preserve group cohesion. This study assessed the form and function of reconciliation between free-ranging female baboons, Papio cynocephalus ursinus, in the Okavango D elta of Botswana. The rate of interaction between former opponents was higher during the minutes that followed conflicts than in the days that preceded or followed conflicts. Baboons reconciled vocally, grunting quietly to their former opponents after conflicts ended. G runts after conflicts facilitated infant handling. Females were particularly likely to reconcile with highranking opponents, the mothers of young infants and related mothers of older infants. R econciliation had no consistent effect upon the rate of interactions during the days that followed conflicts. This study is the first to demonstrate that primates reconcile vocally and the first to assess the long-term consequences of reconcilation in a naturalistic setting.
\end{abstract}

(C) 1996 The A ssociation for the Study of A nimal Behaviour

M onkeys and apes tend to approach and interact non-aggressively with their former opponents at much higher rates immediately after conflicts than at other times. De W aal (1986) first suggested that peaceful contacts after conflicts are a form of reconciliation that enable former opponents to resolve conflicts and repair social relationships damaged by conflict. A Ithough reconciliation has been most systematically studied and widely documented among primates (reviewed by K appeler \& van Schaik 1992), anecdotal accounts of reconciliation also exist for a handful of other taxa (Cords $\&$ Thurnheer 1993). Indeed, if reconciliation does serve to resolve conflicts and repair relationships damaged by conflict, then we might expect it to be common in species that form stable and cohesive social groups.

M ost analyses of reconciliation behaviour have focused upon the form and frequency of events after conflicts; only three have evaluated the

Correspondence: J. B. Silk, D epartment of A nthropology, University of California, Los A ngeles, CA 90095, U.S.A. (email: silk@anthro.sscnet.ucla.edu). D. L. Cheney and R. M. Seyfarth are at the U niversity of Pennsylvania, Philadelphia, PA 19104, U.S.A. actual consequences of post-conflict affiliative behaviours for the participants. In long-tailed macaques, $\mathrm{M}$ acaca fasicularis, the rate of scratching, body shaking and self-grooming by victims increases after conflicts, a sign that victims experience higher levels of sympathetic arousal after conflicts than they do at other times (A ureli et al. 1989; A ureli \& van Schaik 1991). The rate of these behaviour patterns declines more quickly to baseline levels, however, if opponents reconcile than if they do not do so. This result suggests that reconciliation relieves the victims' stress after conflicts. Reconciliation also reduces the probability that the former aggressor will resume its attack upon the victim (A ureli \& van Schaik 1991).

Cords (1993) artificially provoked conflicts between pairs of juveniles in another captive group of long-tailed macaques and evaluated the effect of reconciliation upon the latency to resume drinking in close proximity. She found that reconciliation significantly reduced the time the subordinate took to resume drinking near the dominant partner and reduced the probability that further attacks would occur. In 
a subsequent experiment using the same group of long-tailed macaques, Cords \& Thurnheer (1993) assessed whether the partners' value to one another influenced their tendency to reconcile. During the baseline phase of the experiment, they established the tendency of each pair of monkeys to reconcile and then feed together after artificially provoked conflicts. In this phase of the experiment, dominant animals normally prevented subordinates from feeding near them. Then, in the training phase of the experiment, the monkeys were able to obtain access to food only if they learned to cooperate, i.e. managed to feed simultaneously in close proximity. In the post-training phase of the experiment, the monkeys' responses to artificially provoked conflicts were monitored again, except that in this case monkeys were unable to obtain access to food unless they cooperated. Cords and Thurnheer found that the tendency to reconcile increased after the monkeys were trained to cooperate with their partners, suggesting that monkeys are more likely to reconcile with partners who are valuable to themselves.

Finally, field playback experiments on baboons, Papio cyncocephalus ursinus, have indicated that females were less concerned about becoming the target of redirected aggression from their former opponent if their former opponent had grunted to them after the initial conflict ended than if their former opponent had not grunted to them after the conflict (Cheney et al. 1996). These experiments complement observational studies of baboons and other monkeys that have demonstrated that quiet vocalizations like grunts are a common feature of the behavioural repertoire of many species and often function to facilitate interactions among females of different ranks (reviewed in Cheney et al. 1996).

Here, we used observational data to investigate the form and function of interactions that followed agonistic interactions between adult female members of a group of free-ranging baboons, $P$. cyncephalus ursinus, in the Okavango Delta of Botswana. We focused upon two issues that have received little attention in the reconciliation literature: (1) the role of vocalizations in the post-conflict period and (2) the function of postconflict behaviour patterns. This analysis focused upon interactions between aggressors and their former victims.

\section{METHODS}

\section{Study G roup and Subjects}

The study was conducted in the Okavango D elta of Botswana. One group of baboons at this site has been observed continuously since 1977 by William J. Hamilton III and his colleagues (e.g. Bulger \& Hamilton 1988; Hamilton \& Bulger 1992). The animals are fully habituated to humans on foot and can be identified individually. Subjects were the 19 adult female members of the group. These females were all at least 5 years old, and all were sexually mature. They were ranked in a linear dominance hierarchy that remained stable through the study period. The matrilineal relationships of all individuals born in the group since 1977 are known. In this analysis, we use the term kinship to refer to all females related through the maternal line, i.e. pairs of mothers and daughters, grandmothers and granddaughters, aunts and nieces, and maternal sisters.

\section{D ata Collection and A nalysis}

We conducted 185 10-min focal observations on each of the 19 adult female members of the group over an 11-month period. At the beginning of these interactions, we recorded the focal subject's activity and the identities of all other baboons within a $2-m$ radius. During focal observations, we recorded approaches involving adult and subadult baboons, social interactions with members of all age-sex classes and all vocalizations involving the focal animal. Approaches were scored whenever one baboon moved to within $2 \mathrm{~m}$ of another. A ffiliative interactions included grooming, presenting for grooming, touching and embracing. We recorded infant handling when one baboon picked up, touched, manually inspected, held or briefly carried another female's infant. Supplants occurred whenever one female approached another individual and that individual moved away. Aggressive behaviour patterns included head bobs, eye threats, vocal threats, lunges, chases, bites and attacks.

All focal and post-conflict data were recorded directly onto a hand-held PSION computer and then transferred to a portable computer for editing, tabulation and analysis. Whenever we observed an adult female initiate an aggressive interaction (threat, chase or attack) towards 
another female during a focal sample or ad libitum, we immediately began a focal sample on the aggressor. The post-conflict sample was initiated as soon as the original bout of aggression ended. If aggression resumed during the postconflict period, the sample was extended for another $10 \mathrm{~min}$. Because aggressive interactions did not occur at high rates and because two, and sometimes three, observers were usually present, we were able to conduct post-conflict observations after nearly all aggressive interactions that we saw between females. D uring these post-conflict observations, we recorded the same information that we recorded during standard focal observations. We conducted 514 post-conflict observations, which were restricted to dyadic conflicts between females. The number of post-conflict samples per female varied from 0 to 114, with a median value of 24.5. Only one female was never the subject of a post-conflict sample. She was the lowest-ranking adult female in the group and never initiated aggression against other adult females. The conflicts involved 17 of the 19 females as victims ( median $=27$, range $=0$ to 58 ). The two females who were not involved as victims were a motherdaughter pair who held the top two rank positions. One hundred and forty-nine dyads were represented in the sample of post-conflict observations.

Different methods have been used to determine whether the pattern of interactions that follows conflicts differs from the pattern of interactions observed at other times, but each method has certain shortcomings (reviewed in $\mathrm{K}$ appeler \& van Schaik 1992; Cords 1993; Veenema et al. 1994). Cords (1993) concluded that 'In principle these problems could be avoided if one established a baseline interaction rate for a particular dyad at a particular time, but this would require a density of data exceeding that of any study to date' (page 216). K appeler \& van Schaik (1992) also advocated the use of baseline levels of interaction.

Following these recommendations, we used the large body of focal observations that we collected to establish the baseline rate of interactions between a given pair of opponents immediately before and after each conflict. Post-conflict samples provided data on the rates of behaviour in each dyad immediately after aggression occurred. To compare these rates with the baseline rates of behaviour patterns for the same individ- uals in the days that preceded and followed conflicts, we identified all focal observations that were conducted on the participants (the aggressor and the victim) during a specified period of time before and after the post-conflict observation. Then we tabulated all interactions between the aggressor and the victim during these observations. We initially grouped the baseline data into three blocks of time preceding and following conflicts (1 day, 1-3 days, 1-10 days before and after conflicts). We tabulated and separately analysed events that occurred before and after conflicts. We found that all three blocks of time produced similar results, so we report here only the results of analyses based upon events that occurred 1-10 days before and 1-10 days after each post-conflict sample. F or each post-conflict sample, we conducted on average 13.8 focal samples on the aggressor and victim in the 10 days that preceded the conflict and 13.7 focal samples on the same pair of females during the 10 days that followed the conflict.

To obtain the rate of interaction for each dyad, we computed the number of times that the two females approached, vocalized or interacted with one another during baseline and postconflict periods, and divided these sums by the number of hours in which the two females were observed during the same periods. (Parallel analyses based upon the number of samples in which particular types of interactions were observed, i.e. one-zero sampling, produced very similar results.)

We did not restrict the analysis to observations that were made at the same time of day as the post-conflict sample, because we found little variation in pattern of activity or numbers of animals in proximity during the hours when the data were collected (0700-1300 hours). We did not take the distance between former opponents into account in our analysis of baseline data as suggested by K appeler \& van Schaik (1992), who were concerned that the rates of all types of behaviour would be inflated during the post-conflict period because former opponents were in proximity to one another. Preliminary analyses of our data indicated, however, that the rates of behaviour after conflicts between former opponents were not uniformly elevated in the post-conflict samples: rates of infant handling and affiliation among females were actually lower during the post-conflict period than during baseline samples. 


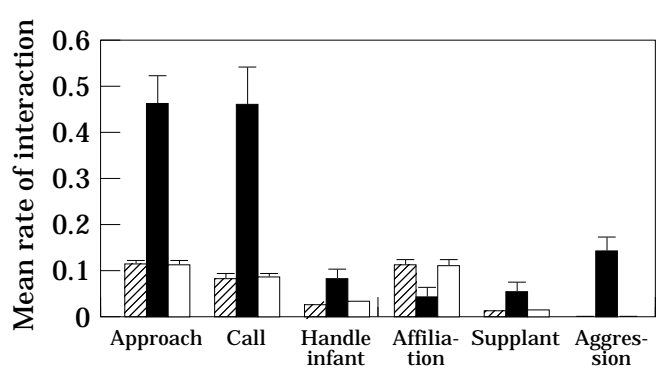

Figure 1. The mean + se rate of approaches, grunts and social interactions involving aggressors and their victims during the 10 days before each conflict $(\square)$, during a 10-min period immediately following each conflict ( $\square$ ) and during the 10 days after $(\square)$ each conflict ( $N=146$ dyads).

\section{RESULTS}

\section{R ates of Interaction}

If female baboons reconcile with their opponents after conflicts, then the rate of nonaggressive interactions between former opponents should be higher during the post-conflict samples than during baseline samples. We were able to compare the rate of interactions before conflicts, during the post-conflict period and after conflicts for 146 dyads. The number of conflicts per dyad ranged from 1 to 12 .

The average rates of interactions between former opponents in the 10 days that preceded conflicts, immediately after conflicts and in the 10 days that followed conflicts are shown in Fig. 1. The rates of approaching and grunting increased during the post-conflict period. Females approached and grunted to their former opponents significantly more often during the post-conflict period than during baseline periods (Wilcoxon matched-pairs signed-ranks test: approach: before versus post-conflict, $\mathrm{z}=-6.06, \mathrm{P}<0.0001,19$ ties; post-conflict versus after, $z=-6.15, P<0.0001,17$ ties; call: before versus post-conflict, $z=-6.05$, $P<0.0001,48$ ties; post-conflict versus after, $z=-5.59, P<0.0001,39$ ties).

In contrast, rates of infant handling and other forms of affiliation among females were generally depressed during the post-conflict period. Although the rate of infant handling averaged across all dyads was higher during the postconflict period, this was not the general pattern. F or most dyads, the rate of infant handling was lower during the post-conflict period than it was before conflicts $(z=-2.05, P=0.0202,89$ ties) or after conflicts ( $z=-1.67, P=0.0473,86$ ties). In this case, the large numbers of ties means that in most dyads, there was no infant handling at any time. The rate of affiliation between former opponents during the post-conflict period was significantly lower than the rate of affiliation before conflicts ( $z=-7.01, P<0.0001,40$ ties) or after conflicts $(z=-7.01, P<0.0001,40$ ties). This result was strikingly uniform across dyads: there were only eight dyads in which the rate of affiliation was higher during the post-conflict period than during the days before or after conflicts.

The post-conflict period was not always a peaceful interlude for females; aggressors sometimes harassed their former victims during the post-conflict period. The rate of aggression was significantly higher during the post-conflict period than it was before conflicts $(z=-5.40$, $P<0.0001,90$ ties) or after conflicts $(z=-5.40$, $P<0.0001,90$ ties). The large number of ties is due to the fact that in many dyads there was no aggression during the baseline or post-conflict period. The rate of supplants during the postconflict period did not differ significantly from the rate of supplants before $(z=-0.03, P=0.4867$, 62 ties) or after conflicts $(z=-0.45, P=0.3251$, 54 ties).

One problem with this analysis is that not all females contributed equally to the data set. High-ranking females were involved in more conflicts with more partners than were lowranking females. We attempted to address this problem by comparing the rate of interactions before conflicts, during the post-conflict period and after conflicts for each female with each of her opponents. These analyses were limited to 14 females who were involved in conflicts with at least five lower-ranking females. Although many comparisons did not produce significant results, the pattern of nearly all of the results was consistent with the pattern based upon the full sample.

The dyadic analysis also did not take into account that differences existed in the baseline rates of interaction between some females. These females generally interacted at higher rates with kin than with non-kin, and at higher rates with the mothers of young infants than with other females. When these factors were controlled in the analysis of differences in the rate of interactions between 
the baseline and post-conflict period, the results remained unchanged.

\section{The Immediate $E$ ffects of R econciliatory B ehaviours}

The high rates of approaches and grunts between aggressors and their former victims during post-conflict periods suggests that one or both of these behaviour patterns may serve reconciliatory functions. Our field playback experiments suggest that approaches by dominant aggressors to their former victims do not serve reconciliatory functions unless they are accompanied by a grunt from the aggressor (Cheney et al. 1996). To assess the effectiveness of approaches and grunts immediately after conflicts, we evaluated the sequence of events that followed approaches during post-conflict samples. We identified all approaches by former opponents and tabulated all events that occurred within $180 \mathrm{~s}$ of the approach. We excluded approaches that took place within $3 \mathrm{~min}$ of the end of post-conflict samples because the data did not provide a full record of the events that followed these acts.

A ggressors approached their former victims 135 times during post-conflict samples. One or both of the females grunted in 51 (38\%) of all approaches. In most cases, only the aggressor called (35/51, $69 \%$ ), but a few approaches occurred in which only the victim called $(\mathrm{N}=9,18 \%)$ or both females grunted to one another $(\mathrm{N}=7,14 \%)$. Victims approached their former aggressors 42 times during post-conflict samples. In 13 (31\%) of these approaches, one or both of the females grunted. $U$ sually, the victim grunted as she approached her former aggressor (10/13, 77\%), but once the aggressor grunted as her victim approached (8\%), and twice both females grunted (15\%).

F emales usually grunted to their former victims shortly after they approached them. Although the average number of seconds that elapsed between the aggressor's approach and grunt was $157 \mathrm{~s}$ $(\mathrm{N}=42$; range 7-588 s), the modal length of the interval was only $8 \mathrm{~s}$. N early half of the calls were given within the first $90 \mathrm{~s}$. When victims approached their former aggressors and grunted to them, the interval between the approach and the grunt was also short $(\mathrm{N}=10$; range $=2-68 \mathrm{~s}$, mode $=3 \mathrm{~s}$ ). In 34 of the 42 cases $(81 \%)$ in which the aggressor approached her former victim and then grunted, the grunt preceeded all other types

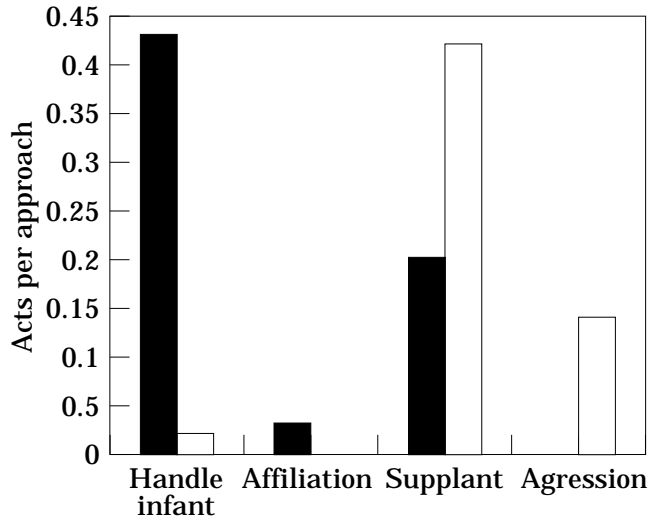

Figure 2. Events following approaches by aggressors to their former victims are compared when only the aggressor called ( $\square$ ) and when neither party called ( $\square$ ). Calls by the aggressor facilitated infant handling and inhibited supplants and aggression.

of interactions. N early all exceptions to this rule $(7 / 8=88 \%)$ involved cases in which the aggressor approached her former opponent, supplanted or attacked her, and then grunted.

Grunts generally seemed to facilitate infant handling and inhibit hostile interactions. Aggressors were more likely to handle their former victim's infants if they grunted after they approached their former victims than if they did not call (Fig. 2). In all of these cases, it was the aggressor that handled her former victim's infant. A ggressors handled their former victims' infants only twice when they did not call, and in both cases the infants were out of contact with their mothers. A ffiliative interactions were rare, and occurred only when the aggressor grunted after approaching her former victim. If aggressors grunted after they approached their former victims, they were less likely to supplant or harass them. There were not enough interactions $(\mathrm{N}=6)$ after approaches by victims to assess the effectiveness of grunts in this context.

To determine whether these patterns were consistent among females, we also examined the sequence of events following approaches by each of the females. We compared the number of interactions that followed approaches in which only the aggressor called with number of interactions that followed approaches in which neither female called. In each case, the number of interactions was divided by the number of 
approaches to correct for differences in the number of approaches with and without calls. We were able to make these comparisons for only 9 females; the others did not approach their former victims during post-conflict periods ( $N=6$ females), did not grunt to their former victims ( $\mathrm{N}=2$ females) or grunted to their former victims in every case ( $\mathrm{N}=1$ female). The frequency of infant handling was significantly higher when females grunted to their former victims after they approached than when they remained silent $(z=-1.75, P=0.0398,4$ ties). G runting consistently reduced the frequency of aggression ( $z=-1.83, \quad P=0.0353,5$ ties) and tended to reduce the frequency of supplants $(z=-2.53$, $P=0.0585,1$ tie). $N$ one of the females compared in this analysis participated in affiliative interactions with their former victims after they approached them.

These results provide a basis for establishing an operational definition of reconciliation in this group. A ggressors often approached and grunted to their former victims after conflicts. G runting substantially increased the probability that the aggressor would handle her former victim's infant during the post-conflict period, and approaches without grunts by the aggressor did not facilitate these kinds of interactions. G runts also reduced the chance that other forms of aggression or competition would occur. A lthough females rarely engaged in grooming during the post-conflict period, grooming only occurred when the aggressor grunted after she approached. Thus, for the remainder of this analysis we will consider conflicts to have been reconciled only if the aggressor grunted to the victim sometime during the post-conflict period.

\section{V ariation in the Tendency to R econcile}

A ggressors grunted to their former victims during the post-conflict period in $13 \%$ of all cases. A ggressors reconciled with some of their victims after all of their conflicts, however, and never reconciled with others. Variation in the tendency to reconcile with particular partners after conflicts may be linked to the nature of the relationship between the aggressor and her victim. Because reconciliation facilitated non-aggressive interactions immediately after conflicts, we might expect females to reconcile with those with whom they are most interested in interacting. We might also expect females to reconcile selectively with those who would be valuable allies in coalitions (Cords \& Thurnheer 1993), and therefore to reconcile at high rates with females who rank just below themselves or with high-ranking females. In most female-bonded species like baboons, females spend much time near their female relatives and selectively support their kin in agonistic conflicts (Silk 1987). Thus, females might be expected to reconcile with kin at relatively high rates (K appeler \& van Schaik 1992). Finally, female baboons are greatly attracted to mothers of newborn infants and are eager to handle newborns (Seyfarth 1976; A Itmann 1980). If females reconcile selectively with the mothers of infants that they want to handle, then the age of the victim's infant may influence the tendency to reconcile.

\section{Dominance rank of participants}

We computed the rank difference between aggressors and their victims by subtracting the aggressor's dominance rank from the victim's dominance rank. Thus, small, positive rank differences characterize females who are close in rank. Females had a small but significant tendency to reconcile more often with females that were close in rank to themselves $\left(r_{s}=-0.1484, P=0.035\right.$, $\mathrm{N}=149$ dyads). The effects of kinship and rank distance were confounded in this correlation because related females occupied adjacent ranks. When dyads composed of related females were removed from the analysis, the correlation coefficient was substantially reduced $\left(r_{s}=-0.1133\right.$, $P=0.091, N=140$ dyads). To ensure that these patterns were consistent between females, we also computed separate correlations for each of the 15 adult females who had at least three victims; one was significantly negative, 12 were nonsignificantly negative, and two were nonsignificantly positive. The probability of obtaining 13 negative correlations by chance alone is quite small (Binomial test: $P=0.004$ ).

A though aggressors outranked their victims in every case, the rank of the victim had a significant effect upon reconciliation. Females reconciled more often with high-ranking victims than with low-ranking victims $\left(r_{s}=-0.2048, P=0.008\right.$, $\mathrm{N}=140$ dyads, kin excluded). Although these correlations are significant, the victim's rank accounts for only $4 \%$ of the variation in the tendency to reconcile. As before, we also 
computed separate correlations for each of the 15 adult females who had at least three victims; 12 were non-significantly negative, one was exactly zero, and one was significantly positive. The probability of obtaining 12 negative correlations by chance alone is small (Binomial test: $P=0.018$ ). The aggressor's rank did not influence the tendency to reconcile with former opponents $\left(r_{S}=-0.0649, \quad P=0.220, \quad N=140\right.$ dyads, kin excluded).

\section{Infant age}

To assess the effects of infant age upon reconciliation, we computed the age of the victim's infant at the time of each conflict. Infants were classified as young (1-90 days old), old (91-365 days old) or other. The last category includes nulliparous females and females whose youngest offspring was more than one year old. Overall, females reconciled in $38 \%$ of their conflicts with mothers of young infants, $12 \%$ of their conflicts with mothers of older infants and $6 \%$ of their conflicts with other females.

In a few cases, we were able to compare the tendency to reconcile in a particular dyad under different conditions, such as when the victim had a small infant and when she had an older infant. Because both infant age and kinship may influence the tendency to reconcile, the analyses that follow are restricted to dyads of unrelated females. F or 13 dyads, we were able to compare the proportion of conflicts reconciled when the victim had a small infant and when she had no offspring under one year of age. $F$ emales reconciled more frequently with unrelated mothers of young infants than with females without infants ( $z=-2.204, P=0.0126,5$ ties). F emales also reconciled more frequently with unrelated mothers of young infants than unrelated mothers of older infants $(z=-1.82, \quad P=0.0346,6$ ties, $N=19$ dyads). There were no significant differences in the proportion of conflicts reconciled with unrelated mothers of older infants and unrelated females who had no infants, largely because females in these two groups rarely reconciled $(z=-1.07$, $\mathrm{P}=0.1425,12$ ties, $\mathrm{N}=15$ dyads).

To determine whether these patterns were consistent among females, we computed the average rate of reconciliation with unrelated mothers of young infants, older infants and females without infants separately for each female. M ost females

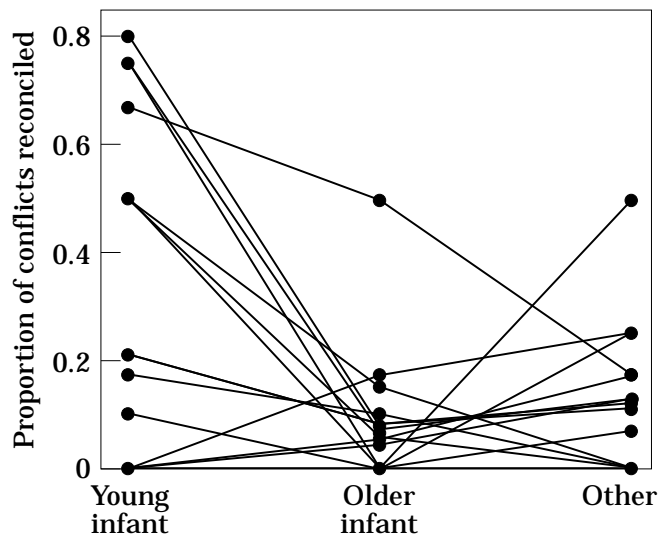

Figure 3. The average proportion of conflicts reconciled with mothers of young infants, mothers of older infants and females who have no infants is shown for individual females. Lines connect points that represent average values for individual females.

reconciled at higher rates on the average with mothers of young infants than with mothers of older infants $(z=-2.58, P=0.0049,0$ ties $)$ or with females without infants $(z=-2.24, P=0.0127$, 1 tie; Fig. 3). The average proportion of conflicts reconciled with mothers of older infants and mothers without infants did not differ significantly $(z=-0.84, P=0.2009,3$ ties).

\section{K inship}

F emales reconciled $31 \%$ of their conflicts with kin and $12 \%$ of their conflicts with non-kin. The influence of kinship was difficult to assess systematically because only six females were involved in conflicts with both lower-ranking kin and nonkin. F or these six females, there was no significant difference in the average proportion of conflicts reconciled with kin and non-kin $(z=0.67$, $P=0.2501,1$ tie).

Although these data suggest that kinship did not affect the tendency to reconcile, some evidence suggested that kinship and infant age jointly influenced the tendency to reconcile. Overall, females reconciled $35 \%$ of all conflicts with related mothers of older infants and $10 \%$ of all conflicts with unrelated mothers of older infants. Females reconciled more often, on average, with related mothers of older infants than with unrelated mothers of older infants $(z=-1.83, P=0.0340$, 1 tie). There were not enough data to assess the 
impact of kinship on reconciliation with mothers of younger infants or females without infants.

\section{The L ong-term F unction of R econciliatory Acts}

If reconciliation repairs social relationships damaged by conflict and helps to maintain social relationships, females who reconciled after conflicts should engage in higher rates of affiliative interactions and lower rates of competitive and agonstic interactions with one another in the days that followed conflicts than females who did not reconcile.

To evaluate this hypothesis, we classified each conflict as reconciled or unreconciled. Then, for each dyad we tabulated the average rate of interactions that occured during the 10-day period that followed reconciled conflicts and unreconciled conflicts. We were able to compare the average rate of interactions after reconciled and unreconciled conflicts for 44 dyads, representing 13 different aggressors and 15 different victims. There were no consistent differences in the rate of approaches ( $z=-0.97, P=0.1672,3$ ties), grunts $(z=-0.040, P=0.3454,5$ ties), infant handling $(z=-1.22, \quad P=0.1105,19$ ties), affiliation $(z=$ $-1.39, \quad P=0.0826,7$ ties), or supplants $(z=-0.36, P=0.72,34$ ties) in the days that followed reconciled and unreconciled conflicts among these dyads. Surprisingly, the rate of aggression was significantly higher following reconciled conflicts than following unreconciled conflicts $(z=-1.95, P=0.0256,13$ ties). The removal of the six dyads composed of related females from the analysis did not alter the results.

To ensure that these patterns were consistent across females, we also computed the average rate of interactions for each of the females with all of their partners. All of the results were consistent with the results based upon individual dyads. The exclusion of related dyads did not alter these results.

\section{DISCUSSIO N}

\section{The $R$ ole of $V$ ocalizations in R econciliation}

During the post-conflict period, the rates of both approaches and grunts were elevated over baseline rates. Grunts by dominant females to their former victims facilitated infant handling between former opponents in the minutes following conflicts, much as grunts by dominant females facilitated affiliative interactions and infant handling in the absence of conflicts (C heney et al. 1996). Females virtually never engaged in nonaggressive interactions with their former victims during the post-conflict period unless they grunted to their victims as they approached.

This result has two important implications. $F$ irst, it suggests that approaches by themselves do not serve a reconciliatory function but instead must be accompanied by grunts for reconcilation to occur. This interpretation is supported by playback experiments (Cheney et al. 1996) that showed that grunts after conflicts restored former opponents' relationships to baseline levels of tolerance, but approaches alone did not have the same effect. Second, it suggests that grunts are a necessary precursor to other types of non-aggressive interactions after conflicts.

Only two other studies have included vocalizations in the analysis of reconciliation (de W aal \& Johanowicz 1993; Watts 1995), which may be an important omission if other species reconcile vocally as baboons do. Juvenile rhesus macaques, M . mulatta, gave affiliative girn vocalizations after conflicts more often when they were housed with stumptailed macaques, M . arctoides, that typically reconcile at high rates than when the rhesus macaques were housed with conspecifics that typically reconcile at low rates (de Waal \& Johanowicz 1993). Female mountain gorillas, Gorilla gorilla gorilla, give non-syllabled grumbles after conflicts with adult males at much higher rates than they do in other contexts. In some studies, reconciliation has not been associated with any particular behaviour pattern. Although overall rates of behaviour were elevated during the post-conflict period, there were no significant changes in the rates of any particular type of interaction (e.g. redfronted lemurs, Eulemur fulvus, K appeler 1993; patas monkeys, Erythrocebus patas, Y ork \& Rowell 1988; long-tailed macaques, A ureli et al. 1989; rhesus macaques, de W aal \& Y oshihara 1983). V ocalizations may play an important role in reconciliation in these cases.

Other studies have concluded that proximity alone is a form of reconciliation (e.g. Y ork \& Rowell 1988; Cords 1993). Our results suggest that approaches alone do not have a reconciliatory function; only approaches with calls do. Because studies that report that proximity alone is 
a form of reconciliation did not examine vocalizations, it is difficult to be certain that this conclusion is correct. Our results suggest that investigation of the role of vocalizations in other species may be profitable.

In baboons, females reconcile vocally and reconciliation facilitates infant handling between former opponents. Former opponents rarely groom or engage in other types of affiliative behaviour during the post-conflict period, even if they have reconciled. $R$ ates of infant handling and affiliation after conflicts are lower than than they are during baseline periods for nearly all dyads. This pattern contrasts with that seen in some other species: grooming and other forms of affiliation occur more often or sooner after conflicts than they do during matched control samples (reviewed in K appeler \& van Schaik 1992). It is not clear why baboons differ from other species in this respect.

\section{The $F$ unction of $R$ econciliation}

The data provide several insights about the function of reconciliation among female baboons. First, reconciliation facilitates non-aggressive interactions, particularly infant handling, and reduces aggression among former opponents in the minutes that follow conflicts. These results are consistent with previous analyses that suggest that reconciliation reduces tension, increases tolerance and reduces further aggresssion between former opponents in the period immediately following conflicts (A ureli et al. 1989; A ureli \& van Schaik 1991; Cords 1993; Cheney et al. 1996).

Second, female baboons reconcile selectively. They are particularly likely to reconcile with the mothers of young infants and with related mothers of older infants. The victim's rank may also influence reconciliatory tendencies to some extent. These results are consistent with the hypothesis that individuals are particularly likely to reconcile with partners that are valuable or attractive to them in other social contexts (Cords \& A ureli 1993; Cords \& Thurnheer 1993), although they do not reveal why females have such preferences.

Third, the long-term effects of reconciliation on social relationships are unclear. $F$ or these female baboons, we could find no persistent effect of reconciliation on the rate of approaches, vocalizations or social interactions between former opponents during the 10 days that followed conflicts. $N$ egative results like these are always difficult to interpret. R econciliation may, for example, influence the nature or quality of interactions in ways that were too subtle to detect in the present analysis. Also, high rates of interaction with valuable partners, such as kin and the mothers of young infants, may be sustained by high rates of reconciliation, even though we found no direct link between reconcilation and the rates of interaction that followed particular conflicts. Finally, reconciliation with former opponents may be only one of a number of different ways that individuals repair and preserve their social relationships. Pairs who did not reconcile after conflicts might have reconciled later, reconciled with their opponent's kin (Cheney \& Seyfarth 1989; Judge 1991) or engaged in other kinds of affiliative contacts that serve similar functions.

The only other report of the long-term effects of reconciliation is an experimental study of reconciliatory tendencies among long-tailed monkeys who learned to cooperate (Cords \& Thurnheer 1993). Changes in the rate of reconciliation did not affect other aspects of the monkeys' social relationships in the days that followed the experimental manipulations, and the authors concluded that the increase in the rate of reconciliation was not associated with an increase in general compatibility among partners.

\section{ONCLUSIONS}

In baboons, reconciliation facilitates nonaggressive interactions between former opponents in the minutes that follow conflicts. For these females, reconciliation seems to be a means to an immediate end, particularly handling a former opponent's newborn infant, and aggressors reconcile selectively with the mothers of newborns. Similarly, young long-tailed macaques selectively reconcile with valuable partners. No evidence suggests that reconciliation enhances or preserves social relationships among individuals over time or that reconciliatory behaviour is necessary for the maintenance of group cohesion. We urge that future work on primates focus upon vocal elements of reconciliation and explore the longterm effects of reconciliation on the quality of social relationships among individuals.

There is no reason to expect reconciliation to be limited to primates. If reconciliation does help to 
normalize relationships after conflicts, then we might expect reconciliation to occur in other species that form stable and cohesive social groups. There are anecdotal accounts of reconciliation in several non-primate species (e.g. dwarf mongoose, $\mathrm{H}$ elogale undulata rufula; hyaena, $\mathrm{Crocuta}$ crocuta; and lions, $\mathrm{P}$ anthera leo; cited in Cords \& Thurheer 1993). Systematic investigation of reconciliation in these species and others may be profitable.

\section{ACKNOW LEDGMENTS}

We thank the Office of the President and the D epartment of Wildlife and $\mathrm{N}$ ational Parks of the R epublic of Botswana for permission to conduct research in the Moremi R eserve. We are grateful to R. Boyd, S. T. S. Boyd, the Longden family, the M cAllister family, J. M. M cN utt, M. Jones, M. K gosiekdae, M. M okopi, C. C. Seyfarth and L. H. Seyfarth for logistical support in the field; and to W. J. Hamilton for making the field camp and demographic records available to us. We thank L. Fairbanks, S. Perry and J. M anson for their comments on the manuscript; $F$. A ureli, $F$. de W aal and M. Cords for their detailed and constructive criticism of the methodology and analysis. This research was supported by grants from the National Geographic Foundation, the Research Foundation of the U niversity of Pennsylvania, the Institute for R esearch in Cognitive Science at the U niversity of Pennsylvania, $\mathrm{NIH}$ Grant HD-29433, and $\mathrm{N}$ ational Science F oundation G rant 9213586.

\section{REFERENCES}

Altmann, J. 1980. Baboon M others and Infants. Cambridge, $\mathrm{M}$ assachusetts: $\mathrm{H}$ arvard $\mathrm{U}$ niversity Press.

A ureli, F. \& van Schaik, C. 1991. Post-conflict behaviour in long-tailed macaques (M acaca fasicularis): II coping with uncertainty. E thology, 89, 101-114.

A ureli, F., van Schaik, C. \& van Hooff, J. A. R. A. M . 1989. F unctional aspects of reconciliation among captive long-tailed macaques (M acaca fasicularis). A m. J . Primatol., 19, 39-51.

Bulger, J.\& Hamilton, W. J. 1988. Inbreeding and reproductive success in a natural chacma baboon, Papio cyncephalus ursinus, population. A nim. Behav., 36, 574-578.
Cheney, D. L. \& Seyfarth, R. M. 1989. R edirected aggression and reconciliation among vervet monkeys, Cercopithecus aethiops. B ehavior, 110, 258-275.

Cheney, D. L., Seyfarth, R. M . \& Silk, J. B. 1996. The role of grunts in reconciling opponents and facilitating interactions among adult female baboons. Anim. B ehav., 50, 249-257.

Cords, M. 1993. On operationally defining reconciliation. A m. J. Primatol., 29, 255-267.

Cords, M . \& A ureli, F. 1993. Patterns of reconciliation among juvenile long-tailed macaques. In: J uvenile Primates: Life $\mathrm{H}$ istory, Development, and Behavior (Ed. by M. E. Pereira \& L. A. F airbanks), pp. 271-284, Oxford: Oxford U niversity Press.

Cords, M.\& Thurnheer, S. 1993. Reconciling with valuable partners by long-tailed macaques. B ehaviour, 93, 315-325.

H amilton, W. J. \& Bulger, J. 1992. F acultative expression of behavioral differences between one-male and multimale savanna baboon groups. Am. J . Primatol., 28, 61-71.

Judge, P. D. 1991. Dyadic and triadic reconciliation in pigtail macaques ( $M$ acaca nemestrina). Am. J. Primatol., 23, 225-237.

K appeler, P. 1993. Reconciliation and post-conflict behaviour in ringtailed lemurs, L emur catta and redfronted lemurs, Eulemur fulvus fulvus. A nim. Behav., 45, 901-915.

K appeler, P. \& van Schaik, C. P. 1992. M ethodological and evolutionary aspects of reconciliation among primates. Ethology, 92, 51-69.

Seyfarth, R. M. 1976. Social relationships among adult female baboons. A nim. B ehav., 24, 917-938.

Silk, J. B. 1987. Social behavior in evolutionary perspective. In: Primate Societies (Ed. by B. B. Smuts, D. L. Cheney, R. M. Seyfarth, R. W. W rangham \& T. T. Strunsaker), pp. 318-319, Chicago: U niversity of Chicago Press.

Veenema. H. C., D as, M . \& A ureli, F. 1994. M ethodological improvements for the study of reconciliation. B ehav. P roc., 31, 29-38

de W aal, F. B. M . 1986. Conflict resolution in monkeys and apes. In: Primates: The Road to Self-Sustaining Populations (Ed. by K. Benirschke), pp. 341-350. Berlin: Springer Verlag.

de W aal, F. B. M . \& J ohanowicz, D. L. 1993. M odification of reconciliation behavior through social experience: an experiment with two macaque species. Child D evel., 64, 897-908.

de W aal, F . B. M . \& Y oshihara, D . 1983. R econciliation and redirected affection in rhesus monkeys. B ehaviour, 85, 224-241.

Watts, D. P. 1995. Post-conflict social events in wild mountain gorillas (M ammalia, H ominoidea). I. Social interactions between opponents. Ethology, 100, 139-157.

York, A. D. \& Rowell, T. E.1988. Reconciliation following aggression in patas monkeys, Erythrocebus patas. A nim. B ehav., 36, 502-509. 